\title{
Effectiveness of different coils for endovascular coiling for intractable hepatic encephalopathy caused by a portosystemic shunt
}

\author{
Toru Ishikawa^, Michitaka Imai, Saori Endo, Motoi Azumi, Yujiro Nozawa, Akito Iwanaga, Tomoe Sano, \\ Terasu Honma, Toshiaki Yoshida
}

Department of Gastroenterology, Saiseikai Niigata Hospital, Niigata, Japan

Contributions: (I) Conception and design: T Ishikawa; (II) Administrative support: T Ishikawa, M Imai; (III) Provision of study materials or patients: T Ishikawa, M Imai, S Endo; (IV) Collection and assembly of data: T Ishikawa, M Imai, S Endo, T Sano; (V) Data analysis and interpretation: T Ishikawa, M Imai, S Endo, T Sano; (VI) Manuscript writing: All authors; (VII) Final approval of manuscript: All authors.

Correspondence to: Toru Ishikawa. Department of Gastroenterology, Saiseikai Niigata Hospital, Teraji 280-7, Niigata 950-1104, Japan. Email: toruishi@ngt.saiseikai.or.jp.

Backgrounds Interventional radiology (IVR), including balloon-occluded retrograde transvenous
obliteration (BRTO) and percutaneous transhepatic obliteration (PTO), is performed for patients with
intractable hepatic encephalopathy (HE). However, information on the appropriate coil for endovascular
coiling for preventing recanalization is lacking. This study aimed to compare the different types of coils for
endovascular coiling used in BRTO and PTO for cases of intractable HE.

Methods: This retrospective study included patients who underwent endovascular coiling with BRTO or PTO for HE caused by portosystemic shunts. The number of coils required for complete occlusion was compared among bare, fiber, and hydrogel-coated coils, and the expansion types that close the gap between and inside the hydrogel-coated coils were also compared.

Results: Of 38 patients (age range, 30-86 years), 16 and 22 underwent BRTO and PTO, respectively, using bare (19 patients), fiber (8 patients), and hydrogel-coated coils (10 patients; external expansion type, 4 ; internal expansion type, 6). No significant differences in the size of portosystemic shunts were observed according to the type of coil. The mean number of coils required for complete occlusion varied (bare coils, 19.32; fiber coils, 18.11; hydrogel-coated coils, 10.70). Significantly fewer coils were required for endovascular coiling with hydrogel-coated coils. In the internal expansion type, a mean of 8.5 coils was required for occlusion.

Conclusions: In some patients who underwent portal vein embolization, complete occlusion was not achieved with the scheduled type of coil because of slight expansion of blood vessels due to coil pressure. The findings suggested that hydrogel-coated coils were effective in endovascular coiling for HE caused by a portosystemic shunt, and internal expansion-type hydrogel-coated coils may be effective for the first-line procedure.

Keywords: Balloon-occluded retrograde transvenous obliteration (BRTO); percutaneous transhepatic obliteration (PTO); bare coil; fiber coil; hydrogel-coated coil

Submitted Sep 12, 2021. Accepted for publication Dec 16, 2021.

doi: 10.21037/apm-21-2594

View this article at: https://dx.doi.org/10.21037/apm-21-2594

$\wedge$ ORCID: 0000-0002-5470-9694. 


\section{Introduction}

Hepatic encephalopathy (HE) is a complex condition with multiple causes, each with varying degrees of severity (1). $\mathrm{HE}$ is a crucial point in the natural history of chronic liver disease (2), and morbidity and mortality sharply increase after the onset of HE (3). HE is classified as type $A$ if it occurs in acute liver failure, as type B if there are portosystemic bypasses without intrinsic hepatocellular disease, and as type $\mathrm{C}$ in the presence of cirrhosis and portal hypertension with portosystemic shunts (4).

To date, balloon-occluded retrograde transvenous obliteration (BRTO) and percutaneous transhepatic obliteration (PTO) have been typically selected for interventional radiology (IVR) treatment of esophageal and gastric varices and type $\mathrm{C}$ HE. These treatments can alter the hemodynamics of the portal vein. Early recanalization frequently occurs after PTO; therefore, PTO has been suggested as a supplementary procedure to other treatments or for emergency treatment of varices (5). In the case of HE, PTO has been found to achieve relatively favorable outcomes in cases in which the portosystemic shunt was successfully embolized with embolic materials such as metallic coils (6). Meanwhile, BRTO has been widely used as the first-line treatment for isolated gastric varices. A recent study reported on the use of BRTO in the treatment of HE (7). Regardless of the technique used, efficient occlusion of the lesion is very important.

This study aimed to evaluate different types of coils used for endovascular coiling in BRTO and PTO for cases of intractable $\mathrm{HE}$ in which a pharmacotherapy-resistant portosystemic shunt was present. We present the following article in accordance with the STROBE reporting checklist (available at https://apm.amegroups.com/article/ view/10.21037/apm-21-2594/rc).

\section{Methods}

This retrospective study included 38 patients who underwent endovascular coiling with BRTO or PTO for HE caused by a portosystemic shunt between January 2015 and November 2020. The selection criteria of these 38 patients were as follows: (I) having cirrhosis and a portosystemic shunt; (II) having an intractable overt HE during the administration of rifaximin and lactulose and (III) pre ammonia levels were over $120.00 \mu \mathrm{g} / \mathrm{dL}$ during the administration of rifaximin and lactulose. Patients with acute hepatic failure and/or hepatocellular carcinoma recurrence during follow-up were excluded.

The procedure was performed under general anesthesia in the IVR angiography suite.

In this study, intractable HE by portosystemic shunt were composed splenorenal shunt or paraumbilical venous shunt. The strategy for splenorenal shunt was selected BRTO. Hence, the strategy for paraumbilical venous shunt was selected PTO.

For BRTO, the right internal jugular vein was accessed. A portosystemic shunt was catheterized via the right renal vein using a balloon catheter, and it is inflated to occlude the venous shunt. A contrast agent was subsequently injected upstream/retrogradely to delineate the anatomy of the pericolic portosystemic collaterals. With the balloon inflated to achieve relative stasis of blood, a microcatheter was advanced through the occlusion balloon catheter deep into the shunt before coiling.

Prior to PTO/percutaneous transhepatic sclerotherapy, ultrasonography was performed for all patients in order to determine the best access route to the portal venous system. The procedure was performed on inpatients under conscious sedation using a combination of $25 \mathrm{mg}$ intramuscular hydroxyzine (Atarax P; Pfizer Japan Inc., Tokyo, Japan) and $15 \mathrm{mg}$ pentazocine (Pentagin; Daiichi Sankyo Co., Ltd., Tokyo, Japan) administered $15 \mathrm{~min}$ before treatment. After injection of $10 \mathrm{~mL}$ of $1 \%$ lidocaine (Xylocaine; AstraZeneca K.K., Osaka, Japan) into the peritoneum along the puncture line, percutaneous transhepatic puncture of the intrahepatic branch of the portal vein was performed using an 18-gauge needle under sonographic guidance. A 5-French sheath catheter was then introduced into the portal vein. Direct portography was performed to identify the feeding and draining veins of the gastric varices or shunt veins. The gastric varices or shunt veins often had multiple feeding veins, and a coaxial catheter was inserted into these feeding veins while avoiding the main feeding vein. PTO is usually performed by placing metallic coils in the afferent veins to reduce blood flow.

The clinical background of the patients was analyzed. Bare, fiber, and hydrogel-coated coils as well as the number of coils required for complete occlusion were compared. Furthermore, for hydrogel-coated coils, the external expansion type that closes the gap between the coils and the internal expansion type that closes the space inside the coils were compared and examined. All used coils were randomly chosen during the procedure by the operator (T.I). 
Table 1 Clinical demographics and patient characteristics $(\mathrm{n}=38)$

\begin{tabular}{|c|c|}
\hline Category & Value \\
\hline \multicolumn{2}{|l|}{ Intervention } \\
\hline BRTO & $16(42.1)$ \\
\hline PTO & $22(57.9)$ \\
\hline \multicolumn{2}{|l|}{ Sex } \\
\hline Male & $24(63.16)$ \\
\hline Female & $14(36.84)$ \\
\hline Age (years) & $64.36 \pm 12.70[30-86]$ \\
\hline \multicolumn{2}{|l|}{ Etiology } \\
\hline HBV & $2(5.26)$ \\
\hline $\mathrm{HCV}$ & $8(21.05)$ \\
\hline NASH & $3(7.89)$ \\
\hline $\mathrm{AlH}$ & $1(2.61)$ \\
\hline PBC & $2(5.26)$ \\
\hline AlHPBC & $2(5.26)$ \\
\hline Alc & $20(52.62)$ \\
\hline \multicolumn{2}{|l|}{ Types of coils } \\
\hline Bare & $19(50.0)$ \\
\hline Fiber & $9(23.68)$ \\
\hline Hydrogel-coated & $10(26.32)$ \\
\hline Outer swelling & $4(10.52)$ \\
\hline Inner swelling & $6(15.79)$ \\
\hline Max shunt diameter (mm) & $19.98 \pm 5.04(11.3-30.4)$ \\
\hline Coil number & $16.76 \pm 7.17[5-30]$ \\
\hline
\end{tabular}

Values are presented as $\mathrm{n}(\%)$ or mean \pm standard deviation (range). BRTO, balloon-occluded retrograde transvenous obliteration; PTO, percutaneous transhepatic obliteration; HBV, hepatitis B virus; HCV, hepatitis C virus; $\mathrm{NASH}$, nonalcoholic steatohepatitis; $\mathrm{AlH}$, autoimmune hepatitis; $\mathrm{PBC}$, primary biliary cholangitis; AIHPBC, autoimmune hepatitis-primary biliary cirrhosis; Alc, alcoholism.

\section{Statistical analysis}

Patient characteristics were summarized using means and standard deviations. Wilcoxon's signed-rank test was used to compare the values obtained before and 24 weeks after treatment. Correlations were determined using Pearson's linear regression analysis. All $\mathrm{P}$ values were two-sided, and $\mathrm{P}<0.05$ was considered statistically significant. Continuous variables were compared using Student's $t$-test or Mann-
Whitney U test. Categorical variables were compared using Fisher's exact test. $\mathrm{P}<0.05$ was considered statistically significant. All statistical analyses were performed using EZR version 1.37 (Saitama Medical Center, Jichi Medical University, Saitama, Japan) (8).

\section{Ethics statement}

The study was approved by the Institutional Review Board of Saiseikai Niigata Hospital (No. E18-18) and was conducted in accordance with the principles of the Declaration of Helsinki (as revised in 2013). All patients provided written informed consent.

\section{Results}

The patients' clinical demographics and characteristics are presented in Table 1. A comparative examination of the clinical background of the patients by the type of coil showed no significant differences in age, sex, background liver condition, or the BRTO/PTO ratio (Table 2). However, the mean number of coils required for complete occlusion varied among bare coils (19.32 coils), fiber coils (18.11 coils), and hydrogel-coated coils (10.70 coils). A significantly smaller number of coils was required for patients who underwent endovascular coiling with hydrogel-coated coils. The maximum size of the portosystemic shunt was slightly larger in the hydrogel-coated coil group than that in other groups, but there were no significant differences (Table 2).

A comparison between the internal and external expansion types of the hydrogel-coated coils found no significant differences in age, sex, background liver condition, or the BRTO/PTO ratio. However, the internal expansion type required fewer coils (8.5) for occlusion (Table 3).

After procedure, serum ammonia levels decreased immediately. All patients improved HE symptoms. The mean follow-up period was 21.25 months. There are no recurrences of overt HE.

\section{Discussion}

In chronic liver disease, progression from hepatitis to cirrhosis can lead to portal hypertension. In portal hypertension, changes in hemodynamics cause various health problems, including the formation of gastric and esophageal varices and portosystemic shunts. Progression of portal hypertension further decreases the blood flow from the portal vein to the liver, increasing the risk of reduced 
Table 2 Patient characteristics according to coil

\begin{tabular}{|c|c|c|c|c|}
\hline Category & Bare $(n=19)$ & Fiber $(n=9)$ & Hydro $(n=10)$ & $P$ value \\
\hline Sex & & & & 0.323 \\
\hline Male & 13 (68.42) & $6(66.67)$ & $6(60.00)$ & \\
\hline Female & $6(31.58)$ & 3 (33.33) & $4(40.00)$ & \\
\hline HBV & $1(5.26)$ & $1(11.11)$ & 0 & \\
\hline $\mathrm{HCV}$ & $3(15.79)$ & $4(44.44)$ & $1(10.00)$ & \\
\hline $\mathrm{NASH}$ & $3(15.79)$ & 0 & 0 & \\
\hline $\mathrm{AlH}$ & 0 & 0 & $1(10.00)$ & \\
\hline Alc & $11(57.89)$ & $3(33.33)$ & $6(60.00)$ & \\
\hline Intervention & & & & 0.451 \\
\hline BRTO & $6(31.58)$ & $3(33.33)$ & $7(70.00)$ & \\
\hline PTO & $13(68.42)$ & $6(66.67)$ & $3(30.00)$ & \\
\hline Maximum shunt diameter (mm) & $17.65 \pm 6.71$ & $19.43 \pm 7.29$ & $21.10 \pm 2.80$ & 0.697 \\
\hline Coil number & $19.32 \pm 7.69$ & $18.11 \pm 5.44$ & $10.70 \pm 3.40$ & 0.004 \\
\hline
\end{tabular}

Values are presented as $\mathrm{n}(\%)$ or mean \pm standard deviation. HBV, hepatitis B virus; HCV, hepatitis C virus; NASH, nonalcoholic steatohepatitis; AlH, autoimmune hepatitis; PBC, primary biliary cholangitis; AlHPBC, autoimmune hepatitis-primary biliary cirrhosis; Alc, alcoholism; BRTO, balloon-occluded retrograde transvenous obliteration; PTO, percutaneous transhepatic obliteration.

hepatic reserve. Notable advances in pharmacotherapy for portal hypertension, including the development of rifaximin for HE, were seen in recent years. Advanced pharmacotherapies have been proven effective in treating complications associated with portal hypertension. Meanwhile, it is important to develop radical treatment strategies for the condition. IVR is increasingly used for patients with chronic liver disease, and it is important to provide such patients with minimally invasive treatment.

The principles of IVR treatment for portal hypertension can be broadly divided into embolization therapy for obstructing varices and shunts and decompression therapy for reducing portal vein pressure. For embolization therapy, transjugular retrograde obliteration (TJO) for gastric varices, BRTO, and PTO are predominantly used.

IVR treatment in which shunts will be obstructed can be divided into retrograde and anterograde techniques. Retrograde techniques include BRTO and TJO for gastric varices, whereas anterograde techniques include PTO.
In 1996, Kanagawa et al. (9) reported on BRTO for isolated gastric varices. Since then, BRTO has been recognized as a breakthrough method that is extremely effective in treating isolated gastric varices. Owing to its convenience and favorable treatment outcomes, BRTO has been used as the primary procedure for this condition. The effectiveness of BRTO for HE has also been verified (9).

Hirota et al. (10) classified the hemodynamics of isolated gastric varices into five grades, with grade 1 having the least progression and grade 5 having the most progression; collateral veins that had developed were treated with embolization. Meanwhile, shunts have various subtypes and are anatomically diverse; therefore, the treatment of the condition can be complex. Although the number inflow passages vary, outflow passages can have the main passage as well as a number of bypass passages. Oldamin may leak into the greater circulatory system if multiple outflow passages are present. In such cases, treatment needs to be discontinued. Aside from oldamin, endovascular coiling is 
Table 3 Patient characteristics by hydro coil obliteration according to expansion pattern

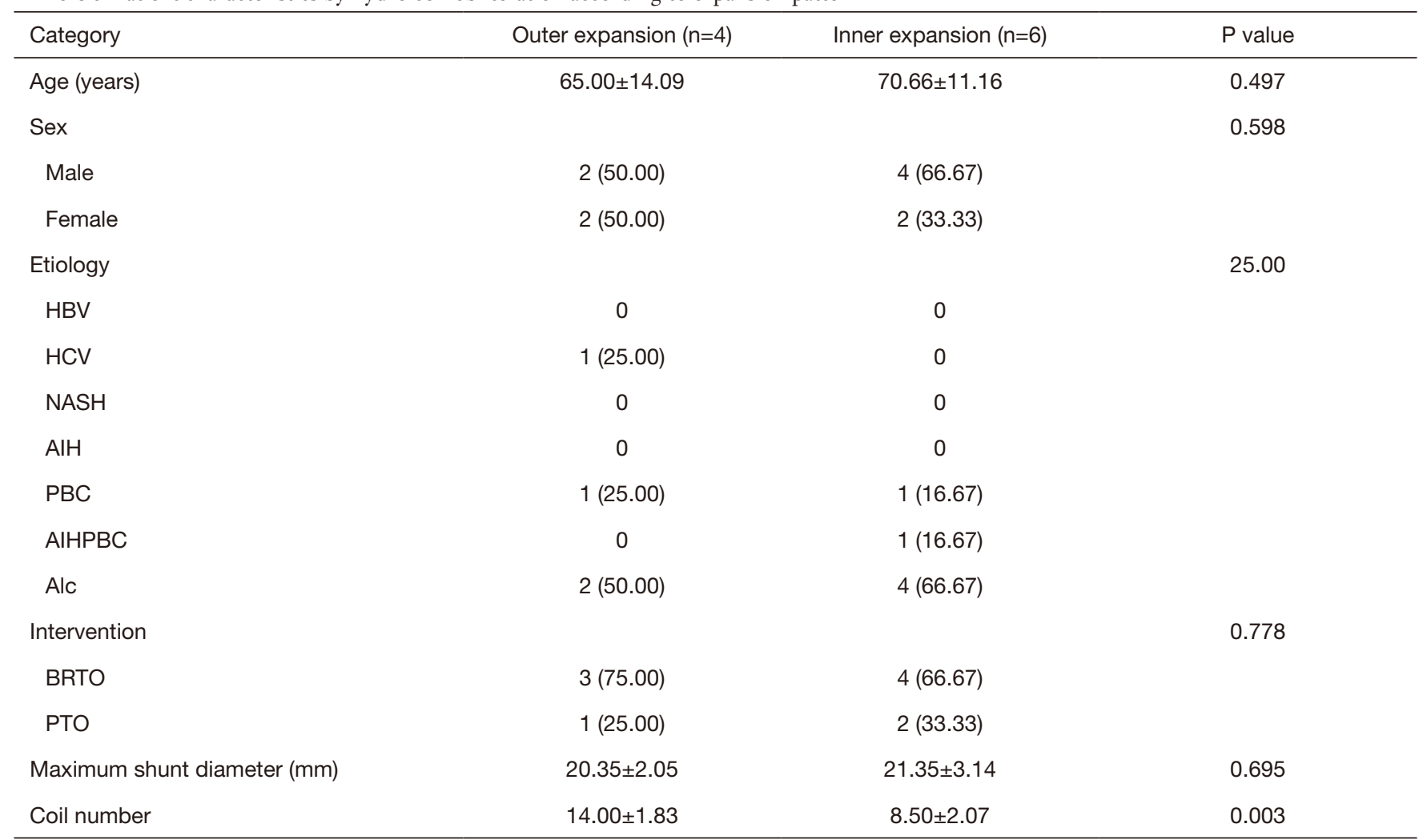

Values are presented as $\mathrm{n}(\%)$ or mean \pm standard deviation. HBV, hepatitis B virus; HCV, hepatitis $\mathrm{C}$ virus; NASH, nonalcoholic steatohepatitis; AlH, autoimmune hepatitis; PBC, primary biliary cholangitis; AlHPBC, autoimmune hepatitis-primary biliary cirrhosis; Alc, alcoholism; BRTO, balloon-occluded retrograde transvenous obliteration; PTO, percutaneous transhepatic obliteration.

also considered effective for embolization.

A study has reported that using a maximum intensity projection of contrast $\mathrm{CT}$ scan in accordance with the portal vein phase prior to BRTO is an effective treatment strategy (11). Furthermore, a transfemoral vein approach was used for BRTO with a catheterization time of $30 \mathrm{~min}$. Meanwhile, Chikamori et al. (12) reported using a transjugular approach in $\mathrm{TJO}$, in which an indwelling catheter was used for 24 hours. In a broad sense, TJO is a type of BRTO. Preoperative diagnostic imaging is key in selecting the approach for TJO.

If a patient has advanced liver dysfunction, portal hypertension leads to an increase in blood flow in the shunt, thereby increasing the volume of blood flow that does not go through the liver and causing HE (also known as shunt encephalopathy). Symptoms of HE can typically be alleviated by conservative treatment with drugs such as branched-chain amino acids and lactulose. However, as the amount of blood flow in the shunt dramatically increases, hyperammonemia persists even if the state of consciousness temporarily improves through conservative treatment. In such a case, the patient is likely to have impaired consciousness.

The most common shunt that can cause HE is a splenicrenal vein shunt, which accounts for $60 \%$ of all cases, followed by the left gastric-renal vein shunt (10\% of the total) and the inferior mesenteric-renal vein shunt (5\%). It is considered that the presence of a portosystemic shunt can cause HE because the shunt leads to encephalopathyinducing substances such as intestinal ammonia to flow directly into the greater circulatory system without going through the liver. HE, which can be explained by this mechanism, is referred to as Inose-type HE. Inose-type HE can only be temporarily improved if internal medical treatment is provided. Long-term treatment of this type of $\mathrm{HE}$ requires procedures on the portosystemic shunt. However, while occluding the portosystemic shunt can lead to improvement of $\mathrm{HE}$, it can also lead to the worsening of 
portal vein pressure, which may result in complications such as exacerbation of gastrointestinal varices, ascites, and portal vein embolism. Thus, treatments and indications must be carefully determined.

PTO was introduced by Lunderquist and Vang (13) in 1974. It has been widely used as an emergency procedure for cases of variceal bleeding. However, PTO, being a new bypass created after the occlusion of the existing pathway, can lead to a recurrence of varices and bleeding. Furthermore, as puncture is performed percutaneously and transhepatically in PTO, this technique is more invasive than other techniques. Nevertheless, as PTO is an anterograde approach, it is useful in understanding hemodynamics through angiography.

An examination of PTO procedures performed for $\mathrm{HE}$ caused by a portosystemic shunt that accompanied portal hypertension and for intractable esophageal/gastric varices showed that the Child-Pugh scores improved 3 months after the procedure (14). The finding suggested that PTO may help maintain hepatic reserve in varying gastric-renal shunts, in intractable HE in which no gastric-renal shunt is present and in which BRTO is ineffective, and in gastric varices. According to Futagawa (15), shunt embolization may be performed when the rate of increase in portal vein pressure following preliminary embolization is up to $50 \%$ to $55 \%$ and the hepatic function has a Child-Pugh score A/ B. According to Tsuruta and Okamoto (16), embolization can be safely performed as long as the portal vein pressure is $\leq 31 \mathrm{cmH}_{2} \mathrm{O}$ after shunt embolization.

However, this study found that complete occlusion was not achieved by BRTO or PTO in certain patients when the scheduled type of coil was used. This was because the blood vessels slightly expanded due to coil pressure in portal vein embolization. Hydrogel-coated coils were originally introduced for the purpose of improving long-term outcomes of intra-aneurysmal embolization for cerebral aneurysm by reducing recanalization. The use of this type of coil in treating the viscera has increased in recent years. In Japan, the use of the Azur ${ }^{\circledR}$ Peripheral Coil System (Terumo Clinical Supply, Gifu, Japan) was approved for the treatment of the viscera in 2012.

Embolization coils can be classified into bare coils, fiber coils, and hydrogel-coated coils. Embolization with bare coils is less effective than other types of coils, but they can be fixed well to the blood vessels. Meanwhile, fiber coils are designed to facilitate embolization with fibers. As the shape and stiffness of the fiber coils are consistent, the coils are unlikely to, or do not, form a clump (i.e., a nest- like shape). Furthermore, as the blood vessels are occluded by fiber-induced embolization, blood flow may be restored if the embolus is lysed by fibrinolysis. Hydrogel-coated coils, which are classified into the internal and external expansion types, are characterized by the expansion of the coating material (i.e., the hydrogel) due to the adsorption of water molecules to the expanded mesh-like structure when the gel comes into contact with blood at a $\mathrm{pH}$ of 7.4. Furthermore, hydrogel does not decompose under physiological conditions (a non-bioactive coil). This study indicates a new strategy for intractable HE for portal hypertension.

Several limitations associated with the present study warrant mention. First, it was a single-center study; thus, there is a possibility of selection bias by area. Second, given the cross-sectional nature of the study, inferring causality was not possible. Third, the recurrence of HE should be examined in the long term.

In this study, although hemodynamics varied among patients, the findings suggested that hydrogel-coated coils were effective in embolization for HE caused by a portosystemic shunt. In particular, internal expansiontype hydrogel-coated coils may be effective as first-line procedure. The hydrogel-coated coil, Azur ${ }^{\circledR}$, was found to be effective, possibly because it can reduce recanalization following endovascular coiling as the expansion of gel increases the filling rate. Future studies should examine a larger number of patients.

\section{Acknowledgments}

We would like to thank Editage (www.editage.com) for English language editing.

Funding: None.

\section{Footnote}

Reporting Checklist: The authors have completed the STROBE reporting checklist. Available at https://apm. amegroups.com/article/view/10.21037/apm-21-2594/rc

Data Sharing Statement: Available at https://apm.amegroups. com/article/view/10.21037/apm-21-2594/dss

Conflicts of Interest: All authors have completed the ICMJE uniform disclosure form (available at https://apm. amegroups.com/article/view/10.21037/apm-21-2594/coif). The authors have no conflicts of interest to declare. 
Ethical Statement: The authors are accountable for all aspects of the work in ensuring that questions related to the accuracy or integrity of any part of the work are appropriately investigated and resolved. The study was approved by the Institutional Review Board of Saiseikai Niigata Hospital (No. E18-18) and was conducted in accordance with the principles of the Declaration of Helsinki (as revised in 2013). All patients provided written informed consent.

Open Access Statement: This is an Open Access article distributed in accordance with the Creative Commons Attribution-NonCommercial-NoDerivs 4.0 International License (CC BY-NC-ND 4.0), which permits the noncommercial replication and distribution of the article with the strict proviso that no changes or edits are made and the original work is properly cited (including links to both the formal publication through the relevant DOI and the license). See: https://creativecommons.org/licenses/by-nc-nd/4.0/.

\section{References}

1. Elsaid MI, Rustgi VK. Epidemiology of hepatic encephalopathy. Clin Liver Dis 2020;24:157-74.

2. Tapper EB, Henderson JB, Parikh ND, et al. Incidence of and risk factors for hepatic encephalopathy in a population-based cohort of Americans with cirrhosis. Hepatol Commun 2019;3:1510-9.

3. Tapper EB, Risech-Neyman Y, Sengupta N. Psychoactive medications increase the risk of falls and fall-related injuries in hospitalized patients with cirrhosis. Clin Gastroenterol Hepatol 2015;13:1670-5.

4. Wright G, Jalan R. Management of hepatic encephalopathy in patients with cirrhosis. Best Pract Res Clin Gastroenterol 2007;21:95-110.

5. Chikamori F, Kuniyoshi N, Kawashima T, et al. Percutaneous transhepatic obliteration for isolated gastric varices with gastropericardiac shunt: case report. Abdom Imaging 2006;31:249-52.

6. Sakurabayashi S, Sezai S, Yamamoto Y, et al. Embolization of portal-systemic shunts in cirrhotic patients with chronic recurrent hepatic encephalopathy. Cardiovasc Intervent Radiol 1997;20:120-4.

7. Numata K, Tanaka K, Kiba T, et al. Use of balloonoccluded retrograde transvenous obliteration with ethanolamine oleate for the treatment of hepatic encephalopathy in a cirrhotic patient with a large spontaneous splenorenal shunt. J Gastroenterol 1998;33:424-7.

8. Kanda Y. Investigation of the freely available easy-touse software "EZR" for medical statistics. Bone Marrow Transplant 2013;48:452-8.

9. Kanagawa H, Mima S, Kouyama H, et al. Treatment of gastric fundal varices by balloon-occluded retrograde transvenous obliteration. J Gastroenterol Hepatol 1996;11:51-8.

10. Hirota S, Matsumoto S, Tomita M, et al. Retrograde transvenous obliteration of gastric varices. Radiology 1999;211:349-56.

11. Ishikawa T, Ushiki T, Mizuno K, et al. CT-maximum intensity projection is a clinically useful modality for the detection of gastric varices. World J Gastroenterol 2005;11:7515-9.

12. Chikamori F, Shibuya S, Takase Y, et al. Transjugular retrograde obliteration for gastric varices. Abdom Imaging 1996;21:299-303.

13. Lunderquist A, Vang J. Transhepatic catheterization and obliteration of the coronary vein in patients with portal hypertension and esophageal varices. N Engl J Med 1974;291:646-9.

14. Ishikawa T, Imai M, Ko M, et al. Percutaneous transhepatic obliteration and percutaneous transhepatic sclerotherapy for intractable hepatic encephalopathy and gastric varices improves the hepatic function reserve. Biomed Rep 2017;6:99-102.

15. Futagawa S. Esophageal varices and portal systemic encephalopathy due to giant collateral and their surgical indications. Acta Hepatol Jpn 1982;23:1494.

16. Tsuruta K, Okamoto A. Closure of the portal-systemic shunt: indication and influence on liver function and on esophageal varices. Jpn J Portal Hypertens 1999;5:83-5.

Cite this article as: Ishikawa T, Imai $M$, Endo S, Azumi $M$, Nozawa Y, Iwanaga A, Sano T, Honma T, Yoshida T. Effectiveness of different coils for endovascular coiling for intractable hepatic encephalopathy caused by a portosystemic shunt. Ann Palliat Med 2022;11(6):1954-1960. doi: 10.21037/apm21-2594 